\title{
Regularization, Recognition and Complexity Estimation Methods of Automata Models of Discrete Dynamical Systems in Control Problem
}

\author{
Anton S. Epifanov \\ Department of Control Problems of Automation Engineering, Institute of Precision Mechanics and Control, Russian Academy of Sciences, \\ Saratov, Russia \\ Email address: \\ epifanovas@list.ru

\section{To cite this article:} \\ Anton S. Epifanov. Regularization, Recognition and Complexity Estimation Methods of Automata Models of Discrete Dynamical Systems in \\ Control Problem. American Journal of Management Science and Engineering. Vol. 2, No. 5, 2017, pp. 106-116. \\ doi: $10.11648 /$ j.ajmse.20170205.14
}

Received: February 26, 2017; Accepted: April 26, 2017; Published: October 23, 2017

\begin{abstract}
In paper are considered laws of functioning of discrete determined dynamical systems and specific processes of functioning of such systems. As basic mathematical model of laws of functioning of systems are used automata models with a fundamentally new extension of these models to models with a countable infinite sets of states. This expansion is possible thanks to the proposed and developed by Tverdohlebov V. A. the mathematical apparatus of geometrical images of automaton mappings. Are presented results of development of regularization methods for partially set automata models of systems based on use of geometrical images of automatons mappings and numerical interpolation methods. Also in paper are considered a problem of complexity estimation of laws in a whole and specific processes of functioning of dynamic systems. For these purpose are used recurrent models and methods and also a specific mathematical apparatus of discrete riv-functions. Is spent classification by complexity estimations of automata models.
\end{abstract}

Keywords: Discrete Dynamical System, Mathematical Model, Automata Model, Geometrical Image of Automaton, Recurrent Model, Interpolation, Discrete Riv-Function

\section{Introduction}

In the theory of experiments with automatons initial base is decoding of a contained of "black box". Initial data is the information on variants of a contained of black box. Under the general scheme of carrying out of experiment to a black box (to contents of a black box) are put influences, reaction to these influences is observed and on these supervision is construction logic conclusions. In control problems the automaton and family of automatons are set and it is required to define, contents of a black box is this allocated automaton or the automaton from the set family. In case of diagnosing it is supposed, that contents of a black box is the element of the set family of automatons and it is required to define what it is the automaton. By E. Mure [1], A. Gill [2], T. Hibbard [3] and other authors solve following problems: criteria of existence of the decision of a problem of recognition of a contained of black box are found; the basic method of construction of experiment on automaton recognition in the set family of automatons, including construction minimum on length of simple unconditional experiment (E. Mure, A. Gill) is developed. Further essentially important expansion of approaches and methods of technical diagnosing was representation of laws of functioning of automatons by geometrical images, i.e. numerical mathematical structures in the form of discrete numerical graphics [4]. If the automatons presented for the decision of control and diagnosing problems in their geometrical images to combine with analytically set curves search and construction of control and diagnostic experiments can be carried out on the basis of the decision of systems of the equations for the geometrical curves set analytically.

In the basic works, containing development of the automata theory, the problem of regularization of automata on the basis of the uniform approach is not considered. There are problems, at which decision used methods assume 
completely set laws of functioning of automatons, but in initial data these laws are presented partially. Fundamental mathematical results on regularization of partially set graphics are presented by classical methods of interpolation of Newton, Lagrange, Gauss, Bessel, spline-interpolation methods etc. Inapplicability of these methods for partially set automatons is connected with the symbolical form of the presentation of automatons by tables, matrixes, graphs, systems of the logic equations, etc. Presentation of laws of functioning of automata by numerical structures, offered and developed by V. A. Tverdokhlebov (see, for example, $[4,5]$ ), allows to use classical methods of interpolation in the automata theory. In paper are developed methods of interpolation for partially set laws of functioning of the automatons, presented by geometrical images.

One of fundamentals, making mathematical models of large-scale systems are the algorithms, realized by system according to its target mission. For an algorithmically solvable class of problems there is an infinite set of algorithms (solving a class of problems), which can be ordered on complexity. Realization of algorithms is generally connected with complexity of algorithm in the system, defining the major indicators: performance, a memory size, reliability, expenditure of energy etc. Number of variants of concept of complexity is sufficiently great and continues to increase in works of many researchers. For example, estimations of algorithms on their belong to NP and P classes (detail review see, for example, in work [6] and one of contemporary papers [7], in which Radoslaw Hofman show, that $\mathrm{P}$ not equal NP), Kolmogorov complexity [8], complexity from below, from above, complexity on the average, bit complexity (one of basic work in this area is [9]), multiplicate complexity, algebraic complexity, there are very large amount of works on asymptotical estimations of complexity (see, for example works $[10,11]$ ), approximation complexity of analysis of graph structures [12,13] and for identifying codes [14] etc.

In the given work with use of the apparatus of geometrical images of automatons [4], it is offered and is investigated the estimation of complexity of laws of functioning of the discrete determined dynamic systems (automatons) on the basis of discrete riv-functions of kind $H^{d, k}(t)=\left[h_{1}^{d, k}(t), h_{2}^{d, k}(t)\right]$, where and $h_{1}^{d, k}(t)$ and $h_{2}^{d, k}(t)$ - the finite discrete determined functions of a kind $h^{d, k}:\{1,2, \ldots, \mathrm{d}\} \rightarrow\{0,1,2, \ldots, \mathrm{k}\}$, defined on initial segment of the natural scale, accepting values from initial segment of the natural scale and for any $t$, where $1 \leq t \leq \mathrm{d}, \mathrm{d}, \mathrm{k} \in \mathrm{N}^{+}, h_{1}^{d, k}(t) \leq h_{2}^{d, k}(t)$. Riv-function is set of such finite discrete determined functions of a kind $h^{d, k}$, which satisfy to a condition: for any $t$, where $1 \leq t \leq \mathrm{d}, \mathrm{d} \in \mathrm{N}$ ${ }^{+}, h_{1}^{d, k}(t) \leq h^{d, k}(\mathrm{t}) \leq h_{2}^{d, k}(t)$. Borders $h_{1}^{d, k}(t) \leq h_{2}^{d, k}(t)$, defined by function $H^{d, k}$ and forming "corridor", are considered as geometrical images of laws of functioning of automatons (at the chosen set of input signals of the automaton and chosen order on set of input sequences). The graph of discrete function $h^{d, k}$, where $h_{1}^{d, k}(t) \leq h^{d, k}(\mathrm{t}) \leq h_{2}^{d, k}(t), 1 \leq t \leq \mathrm{d}$, also is considered as a geometrical image of the automaton. Thus, the class $\Psi\left(H^{d, k}, m\right)$ of the discrete determined automatons, defined by discrete riv-function $H^{d, k}(t)=\left[h_{1}^{d, k}(t), h_{2}^{d, k}(t)\right]$ and chosen number $\mathrm{m}$ - number of input signals of the automaton, consists of $\Theta\left(H^{d, k}(t)\right)=\prod_{t=1}^{d}\left(h_{2}^{d, k}(t)-h_{1}^{d, k}(t)+1\right)$ automatons.

In this work is offered the estimation of complexity of laws of functioning of the discrete determined dynamic systems (automatons) on the basis of geometrical representation of laws and use of discrete riv-functions. Is carried out the analysis of more than 10 million discrete rivfunctions. In clause are considered the riv-functions, containing more of 20 billion of discrete graphs, on which are synthesised laws of functioning of automatons. Specificity of all considered riv-functions is defined. As complexity indicators are considered the minimum and maximum number of states at the minimal automaton from the set of automatons, defined by riv-function.

\section{Geometrical Images of Lows of Functioning of Automatons}

It is known, that the apparatus of continuous numerical mathematics effectively uses infinite sets. In this connection Tverdohlebov V. A. was developed the new approach to construction of models of complex systems and methods of the analysis of such models, which are stated in works [4, 5]. A developed principle is placing of discrete structures on continuous geometrical curves, set analytically. For this purpose instead of next-state function and output function of automaton is considered a automaton mapping, i.e. symbolical mathematical structures of a kind (input sequence, output sequence). Geometrical image $\gamma_{s}$ of laws of functioning (see works $[4,5]$ ) of initial finite determined automaton $A_{s}=(S, X, Y, \delta, \lambda, s)$ with sets of states $S$, input signals $X$, output signals $Y$ and next-state function $\delta: S \times X \rightarrow S$ and functions of outputs $\lambda: S \times X \rightarrow Y$ it is defined on the basis of introduction of a linear order $\omega$ in automata mapping $\rho_{s}^{\prime}=\bigcup_{p \in X^{*}}\{(p, \lambda(s, p))\}$, where $\lambda(s, p)=\lambda\left(\delta\left(s, p^{\prime}\right), x\right)$, at $p=p^{\prime} x$.

Automaton mapping $\rho_{s}$ (set of pairs) is ordered by linear order $\omega$, defined on the basis of an order $\omega_{1}$ on $X^{*}$ and set by following rules:

Rule 1. On set $X$ some linear order $\omega_{1}$ (which we will designate $\prec_{1}$ ) is entered

Rule 2. An order $\omega_{l}$ on $X$ we will extend to a linear order on set $X^{*}$, believing, that

a. For any words $p_{1}, p_{2} \in X^{*}$ with unequal length $\left(\left|p_{1}\right| \neq \mid p_{2}\right.$ |) $\left|p_{1}\right|<\left|p_{2}\right| \rightarrow p_{1} \prec_{1} p_{2}$;

b. For any words $p_{1}, p_{2} \in X^{*}$ for which $\left|p_{1}\right|=\left|p_{2}\right|$ and $p_{1} \neq$ $p_{2}$, their relation in the order of $\omega_{1}$ repeats the relation of the incoincident letters of words, nearest at the left in $p_{1}$ and $p_{2}$. The order $\omega_{2}$ on set of words $Y^{*}$ is similarly defined. 
After introduction on set $X^{*}$ of a linear order $\omega_{l}$, we receive linearly ordered set $\rho_{s}=\left(\rho_{s}^{\prime}, \omega_{1}^{\prime}\right)$, where $\omega_{1}^{\prime}$ - an order on $\rho_{s}^{\prime}$, induced rather $\omega_{1}$ on $X^{*}$. Having a linear order $\omega_{2}$, defined on set $Y$ and having placed set of points $\rho_{\mathrm{s}}$ in system of coordinates $D_{l}$ with an axis of abscisses $\left(X^{*}, \omega_{l}\right)$ and an axis of ordinates $\left(Y, \omega_{2}\right)$, we receive a geometrical image $\gamma_{\mathrm{s}}$ of laws of functioning of initial finite determined automaton $A_{s}=(S, X, Y, \delta, \lambda, s)$. Linear orders $\omega_{1}$ and $\omega_{2}$ allow to replace elements of sets $X^{*}$ and $Y$ by their numbers $r_{1}(p)$ and $r_{2}(p)$ on these orders. As a result are defined two forms of geometrical images, first, as symbolical structure in system of coordinates $D_{l}$, and secondly, as numerical structure in system of coordinates with integer or real positive semiaxes.

\section{The Method of Recognition of Automatons by Their Geometrical Images}

Let automaton $\mathrm{A}_{0}$ is mathematical model of efficient technical system and the family of automatons $\alpha=\left\{A_{i}\right\}_{i \in I}$ represents set I of failures of technical system. We will assume, that these automatons are set by geometrical image $\gamma_{0}$ and family of geometrical images $\beta=\left\{\gamma_{i}\right\}_{i \in I}$. In the developed method of recognition geometrical images $\gamma_{0}$ and $\left\{\gamma_{i}\right\}_{i \in I}$ rely located on analytically set geometrical curve $L_{0}$ and family of analytically set geometrical curves $L=\left\{L_{i}\right\}_{i \in I}$. Then equality $\left\{L_{0}\right\} \cap \bigcup_{i \in I}\left\{L_{i}\right\}=\varnothing$, where $\left\{\mathrm{L}_{0}\right\}$ and $\left\{L_{i}\right\}_{i \in I}$ - sets of points of curves, is defined the decision of a problem of the control with use of simple unconditional experiment.

Definition 3.1. Let $L$ - a geometrical curve and $\Delta-$ a piece on an axis of abscisses, on which the part of curve $L$ (or all curve $L$ ) is defined. This part of a curve we will designate $L$ $(\Delta)$.

Theorem 3.1. Let initial automaton $A_{0}=\left(S, X, Y, \delta, \lambda, s_{0}\right)$ have a geometrical image $\gamma_{0}$, located on curve $L_{0}$ and $\alpha=\left\{A_{i}\right\}_{\in I}$ - a family of initial automatons, where $A_{i}=\left(S_{i}\right.$, $\left.X, Y, \delta_{i}, \lambda_{i}, s_{0_{i}}\right), s_{0_{i}} \in S_{\mathrm{i}}$, and $\left.\beta=\mho_{i}\right\}_{\in I}$ - a family of their geometrical images, located accordingly on curves from family $L=\left\{L_{i}\right\}_{i \in I}$. If $L_{0}(\Delta) \cap L_{i}(\Delta)=\varnothing, i \in I$, is carried out and in a piece $\Delta$ of abscissa axis are defined some points of geometrical image $\gamma_{0}$ and geometrical images from family $\beta$, then piece $\Delta$ contains the decision of a problem of recognition of the automaton concerning of family $\alpha$ by simple unconditional experiment.

The proof. Let $I=\{1,2, \ldots, k\}$. We will present system of equalities $\left\{L_{i}(\Delta)\right\} \cap\left\{L_{j}(\Delta)\right\}=\varnothing, i, j \in I, i \neq j$, as conjunction of separate statements: $\left(\quad\left\{L_{1}(\Delta)\right\} \cap\left\{L_{2}(\Delta)\right\}=\varnothing\right) \&\left(\left\{L_{1}(\Delta)\right\} \cap\left\{L_{3}(\Delta)\right\}=\varnothing \quad\right) \quad \& \quad \ldots \quad \& \quad \&$ $\left(\left\{L_{\mathrm{k}-2}(\Delta)\right\} \cap\left\{L_{k}(\Delta)\right\}=\varnothing\right) \&\left(\left\{L_{\mathrm{k}-2}(\Delta)\right\} \cap\left\{L_{k}(\Delta)\right\}=\varnothing\right)$. If $p \in X^{*}$ on construction of geometrical images as binary relations $p$ is the first coordinate of some points in all geometrical images $\gamma_{i}, i \in I$. From equality $\left.\left\{L_{1}(\Delta)\right\} \cap L_{2}(\Delta)\right\}=\varnothing$, follows, that at any choice $p \in X^{*}$, at which $r_{1}(p) \in \Delta, \gamma_{1}(p) \neq \gamma_{2}(p)$ and the observable behaviour of automaton $A_{1}$ and $A_{2}$ is recognized by simple unconditional experiment. Similar conclusions take place for all equalities from conjunction of separate statements, i.e. all pairs of automatons of a kind $\left(A_{\mathrm{i}}, A_{\mathrm{j}}\right)$, $i, j \in I, i, j \neq I$, are recognized by output sequences on the general input sequence $p$, i.e. by simple unconditional experiment.

On the basis of the theorem 1 is offered the method (with 4 stages) of recognition of the automaton, which laws of functioning are set by the geometrical images, located on analytically set curves.

Stage 1. Construction (choice) of family $L=\left\{L_{i}\right\}_{i \in I}$ of geometrical curves and an arrangement on them of geometrical images of laws of functioning of automatons from family of automatons $\alpha=\left\{A_{i}\right\}_{\in I}$.

Stage 2. For system of equalities $\left\{L_{\mathrm{i}}\right\} \cap\left\{L_{j}\right\}=\varnothing, i, j \in I, i \neq$ $j$, is defined the family of decisions $\left\{\Delta_{i j}\right\}, i, j \in I, i \neq j$.

Stage 3. Piece $\Delta=\bigcap_{i \neq j} \Delta_{i j}$, which on construction satisfies to following conditions, is defined:

1. If $\Delta \neq \varnothing$, then each point of a piece $\Delta$, which is the first coordinate of points of geometrical images of automatons from family of automatons $\alpha$, defines the decision of a problem of recognition of the automaton in family of automatons by simple unconditional experiment.

2. If $\Delta=\varnothing$ then for chosen concrete geometrical curves $L_{\mathrm{i}}$, $i \in I$, and the placing of geometrical images of laws of functioning of automatons on these curves, the decision of a problem of recognition of the automatons in family of automatons by simple unconditional experiment does not exist.

Stage 4. According to conditions $\Delta \neq \varnothing$ or $\Delta=\varnothing$ is defined the concrete decision of a problem of recognition of the automaton in family of automatons by simple unconditional experiment or the conclusion becomes, that for family $\alpha$ of automatons, and the chosen family of geometrical curves $L$ and the chosen arrangement of geometrical images on curves the decision of a problem of recognition of the automaton in family of automatons by simple unconditional experiment does not exist.

\section{The Method of Recognition of Automaton in Pair of Automatons by Geometrical Images}

For automaton recognition in the set family of automatons is offered to represent sequences of the second coordinates of points of geometrical images of automatons by sequences of values of the characteristic functions, reflecting an arrangement of values of elements in initial sequences. On the basis of the analysis of sequences of the characteristic functions, constructed for sequences of the second coordinates of points of geometrical images of automaton, the problem of recognition of the automaton are solved.

Definition 4.1. On set $Y^{*}$ we will define family $\Phi=\left\{\varphi_{y}\right\}_{y \in Y}$ of characteristic functions by a rule: for any $y \in Y$ and any 
sequence $\xi=y_{i_{1}} y_{i_{2}} \ldots y_{i_{k}} \quad \varphi_{y}(\xi)=\varphi_{y}\left(y_{i_{1}}\right) \varphi_{y}\left(y_{i_{2}}\right) \ldots \varphi_{y}\left(y_{i_{k}}\right)$, where $\varphi_{y}\left(y_{i_{j}}\right)=0$, if $y_{i_{j}} \neq y$, and $\varphi_{y}\left(y_{i_{j}}\right)=1$, if $y_{i_{j}}=y$.

The method consists of following stages:

Stage 1. For automaton $A_{1}=\left(S_{1}, X, Y, \delta_{1}, \lambda_{1}, s_{01}\right)$ and $A_{2}=$ $\left(S_{2}, X, Y, \delta_{2}, \lambda_{2}, s_{02}\right)$, making an exclusive class and set in the geometrical images $\gamma_{1}^{d}$ and $\gamma_{2}^{d}$, where $\left|\gamma_{i}^{d}\right|=d, i \in\{1,2\}$, sequences $\xi_{1}^{d}$ and $\xi_{2}^{d}$ the second coordinates of points of geometrical images are constructed.

Stage 2. For everyone $y \in Y$ and for each of sequences $\xi_{1}^{d}$, $\xi_{2}^{d}$ values $\varphi_{y}\left(\xi_{1}^{d}\right)$ and $\varphi_{y}\left(\xi_{2}^{d}\right)$ characteristic function $\varphi_{y}$ are defined.

Stage 3. For everyone $y \in Y$ is defined the least length $\mathrm{r}_{\mathrm{y}}\left(\varphi_{y}\left(\xi_{1}^{d}\right), \varphi_{y}\left(\xi_{2}^{d}\right)\right)$ of not equal initial pieces of sequences $\varphi_{y}\left(\xi_{1}^{d}\right)$ and $\varphi_{y}\left(\xi_{2}^{d}\right)$. The initial piece of length $\mathrm{r}=\min _{y \in Y}$ $\left(\mathrm{r}_{\mathrm{y}}\left(\varphi_{y}\left(\xi_{1}^{d}\right), \varphi_{y}\left(\xi_{2}^{d}\right)\right)\right)$ unequivocally defines the least on length sequence of input signals for simple unconditional experiment on automaton recognition in pair of automatons.

The method is proved by the following theorem.

Theorem 4.1. The problem of recognition of the automaton by simple unconditional experiment in pair of automatons $A_{1}$ and $A_{2}$ where $A_{1}=\left(S_{1}, X, Y, \delta_{1}, \lambda_{1}, s_{01}\right)$ and $A_{2}=\left(S_{2}, X, Y, \delta_{2}\right.$, $\left.\lambda_{2}, s_{02}\right), S_{1} \cap S_{2}=\varnothing$, has the decision in only case when when for some $y \in Y$ characteristic function $\varphi_{y}$ satisfies to condition $\varphi_{y}\left(\xi_{1}^{d}\right) \neq \varphi_{y}\left(\xi_{2}^{d}\right)$ for sequences $\xi_{1}^{d}$ and $\xi_{2}^{d}$ of lengths $d=\sum_{i=1}^{n^{2}-1}|X|^{i}$, where $\mathrm{n}=\left|\mathrm{S}_{1}\right|+\left|\mathrm{S}_{2}\right|$, of the second coordinates of points of geometrical images of automatons $A_{1}$ and $A_{2}$.

\section{Interpolation for Regularization of Laws of Functioning of Automatons}

The choice and application of a method of interpolation by implication correspond to acceptance and realization of a hypothesis, that the method of interpolation, applied to the numerical graphic, representing partially set geometrical image of the automaton, enough precisely regularize points of a geometrical image, i.e. is enough exact regularize partially set laws of functioning of the automaton. Therefore, validity of the results, received with use of the chosen method of interpolation, is shown to a substantiation of correctness of a hypothesis. In the given paragraph methods of a choice of a hypothesis (a choice of a concrete method of interpolation) are investigated and developed for concrete classes of automatons on an example of a choice of more exact method of interpolation from two methods of interpolation: Newton and Lagrange (under the similar scheme also is carried out the analysis of Gauss, Bessel etc. methods). These methods include following stages:

1. Stage. It is defined and obviously is constructed the class $U$ of automatons, in which partially set automatons by method of interpolation of their partial geometrical images regularize to full geometrical images. The set of methods of interpolation are choose for research (in the given chapter the set include Newton's and Lagrange methods).

2. Stage. For interpolation are defined base points (in work for research are considered 2 variants of a choice of base points of interpolation: use as base points tops of geometrical images of autonomous subautomatons and use as base points of interpolation of those tops of geometrical images of laws of functioning of automatons, which are located on the straight lines, parallel to an axis of abscisses).

3. Stage. Choice of the length $d$ of geometrical image, by which partial representation the geometrical image of laws of functioning of the automaton is interpolated.

4. Stage. To base points of interpolation, chosen at a stage 2, are applied Newton's and Lagrange interpolation methods.

5. Stage. Results of interpolation are represented by following numerical indicators:

a. For everyone initial automaton and each method of interpolation is defined the number of correctly restored (regularized) tops of a geometrical image of laws of functioning of the automaton;

b. - For a considered class of automatons and the set length $d$ of geometrical images of laws of functioning of automatons are calculated numerical indicators $n_{d}^{N}$ - number of automatons in a considered class, for which by Newton's method it is correctly restored more points, than by method Lagrange, $n_{d}^{L}-$ number of automatons in a considered class, for which by method Lagrange it is correctly restored more points, than by Newton's method and $\mathrm{n}_{\mathrm{d}}^{\mathrm{NL}}$ - number of automatons in a considered class, for which Newton's and Lagrange methods have identical efficiency.

6. Stage. Choice of function for an estimation of efficiency of methods of interpolation, i.e. for definition of the most effective method in an investigated set of interpolation methods. In paper is offered and used function $F\left(n_{d}^{N}, n_{d}^{L}, n_{d}^{N L}\right)=1-\frac{\min \left(n_{d}^{N}, n_{d}^{L}\right)+n_{d}^{N L}}{\max \left(n_{d}^{N}, n_{d}^{L}\right)+n_{d}^{N L}}$, with the following condition $\mathrm{n}_{\mathrm{d}}^{\mathrm{N}}+\mathrm{n}_{\mathrm{d}}^{\mathrm{L}}+\mathrm{n}_{\mathrm{d}}^{\mathrm{NL}} \neq 0$, where $n_{d}^{N}, n_{d}^{L}$, $n_{d}^{N \mathrm{~L}}$ defined higher in stage 5 , which values allows to compare efficiency of Newton's and Lagrange methods. Properties of function F are presented in [5].

Theorems 5.1, 5.2 contain results of the analysis of efficiency of application of Newton's and Lagrange methods to partially set geometrical images of autonomous subautomatons of automatons from a class of $(4,2,2)$ automatons (more than 67 million initial automatons) at various values of length of an initial piece of a geometrical image.

Theorem 5.1. Let base points of interpolation for partially set geometrical image of length d of each automaton $A=(S, X$, $\left.Y, \delta, \lambda, s_{0}\right)$ from class of $(4,2,2)$-automatons are points of geometrical images of autonomous subautomatons $A_{1}=(S$, $\left.\{0\}, Y, \delta_{1}, \lambda_{1}, s_{01}\right)$ and $A_{2}=\left(S,\{1\}, Y, \delta_{2}, \lambda_{2}, s_{02}\right)$ of automaton 
A. Then for Newton's and Lagrange methods at $\mathrm{d}=30$ in a class of initial $(4,2,2)$ - automatons is true relation $n_{d}^{N}>n_{d}^{L}$ and function $\mathrm{F}$ accepts value $\mathrm{F}\left(\mathrm{n}_{30}^{\mathrm{N}}, \mathrm{n}_{30}^{\mathrm{L}}, \mathrm{n}_{30}^{\mathrm{NL}}\right)=0,65$ (method of interpolation of Newton with an assessment 0.65 more precisely, than a method of Lagrange).

Theorem 5.2. Let base points of interpolation for partially set geometrical image of length d of each automaton $A=(S, X$, $\left.Y, \delta, \lambda, s_{0}\right)$ from class of $(4,2,2)$-automatons are points of geometrical images of autonomous subautomatons $A_{1}=(S$, $\left.\{0\}, Y, \delta_{1}, \lambda_{1}, s_{01}\right)$ and $A_{2}=\left(S,\{1\}, Y, \delta_{2}, \lambda_{2}, s_{02}\right)$ of automaton A. Then for Newton's and Lagrange methods at $\mathrm{d}=254$ in a class of initial $(4,2,2)$ - automatons is true relation $n_{d}^{N}>n_{d}^{L}$ and function $\mathrm{F}$ accepts value $\mathrm{F}\left(\mathrm{n}_{254}^{\mathrm{N}}, \mathrm{n}_{254}^{\mathrm{L}}, \mathrm{n}_{254}^{\mathrm{NL}}\right)=0,14$ (method of interpolation of Newton with an assessment 0.14 more precisely, than a method of Lagrange).

Proofs of theorems 5.1, 5.2 contains, for example, in [5].

\section{Complexity Estimation Methods of Automata Models of Discrete Dynamical Systems}

\subsection{Problem Statement}

The finite determined automaton is set by finite geometrical image of laws of functioning (on a finite section). It is required to define the top and bottom borders for number of states without obvious construction of nextstate function and output function of automaton. The method of the decision of a problem is based on theorems, in which for various configurations of borders of areas on planes and assumptions, that the geometrical image of the automaton is in these areas, the top and bottom estimations for number of states in reduced form of automaton are defined. For each considered configuration of borders of areas on a plane are defined:

a. The maximal number of conditions of a reduced form of the automaton, which geometrical image it is located in borders of considered area on a plane;

b. The minimal number of conditions of a reduced form of the automaton, which geometrical image it is located in borders of considered area on a plane.

The received estimations extend on all automatons, geometrical images are limited by configurations of the top and bottom borders of area on a plane.

Set of configurations of borders of the investigated areas on a plane:

1. The rectangular form of area (the top and bottom borders represent direct, parallel axes of abscisses);

2. A configuration, in which borders are presented by the sequences, defining approach of fundamental mathematical constants $\left(\pi\right.$, e, $\left(\varphi=\frac{1+\sqrt{5}}{2}\right.$ so-called gold section), $\sqrt{2}, \sqrt[3]{2}$, $\ln (2), \ln (10), \zeta(3)=\sum_{x=1}^{\infty} \frac{1}{x^{3}}$, constant of Catalan $C=\sum_{n=0}^{\infty} \frac{\left(-1^{n}\right)}{(2 n+1)^{2}}$, Euler's constants $H^{d, k}=\left[h_{1}^{d, k}, h_{2}^{d, k}\right]$ etc. $)$.

\subsection{Descrete Riv-Functions}

The finite The finite discrete determined riv-functions of a kind $H^{d, k}=\left[h_{1}^{d, k}, h_{2}^{d, k}\right]$, where $h_{1}^{d, k}(t)$ and $h_{2}^{d, k}(t)$ - the finite discrete determined functions of a kind $h^{d, k}:\{1,2, \ldots, \mathrm{d}\} \rightarrow$ $\{0,1,2, \ldots, \mathrm{k}\}$, defined on initial section of the natural scale, accepting values from initial section of a natural scale and satisfying to a condition: for any $t$, where $1 \leq t \leq \mathrm{d}, \mathrm{d} \in \mathrm{N}^{+}$, $h_{1}^{d, k}(t) \leq h_{2}^{d, k}(t)$. Riv-function represents set of such finite discrete determined functions of a kind $h^{d, k}$, which satisfy to a condition: for any $t$, where $1 \leq t \leq \mathrm{d}, \mathrm{d} \in \mathrm{N}^{+}, h_{1}^{d, k}(t) \leq$ $h^{d, k}(t) \leq h_{2}^{d, k}(t)$. The offered name of function represents reduction from an english word river.

Let's give formal definitions.

Definition 6.1. Function $\mathrm{h}^{\mathrm{d}, \mathrm{k}}$ of kind $\mathrm{h}^{\mathrm{d}, \mathrm{k}}:\{1,2, \ldots, \mathrm{d}\} \rightarrow$ $\{0,1,2, \ldots, \mathrm{k}\}$ where, $d, k \in N^{+}$we will name discrete determined integer ( $\mathrm{d}, \mathrm{k}$ )-function (or simple ( $\mathrm{d}, \mathrm{k})$-function).

Definition 6.2. Discrete riv-function $H^{d, k}=\left[h_{1}^{d, k}, h_{2}^{d, k}\right]$, where $h_{1}^{d, k}$ and $h_{2}^{d, k}-(\mathrm{d}, \mathrm{k})$-functions and for any $t$ where $1 \leq$ $t \leq \mathrm{d}, \mathrm{d} \in \mathrm{N}^{+}, h_{1}^{d, k}(t) \leq h_{2}^{d, k}(t)$, we will be name set of such (d, k)-functions $h^{d, k}$, for which the condition is satisfied: for any $t$, where $1 \leq t \leq \mathrm{d}, h_{1}^{d, k}(t) \leq h^{d, k}(t) \leq h_{2}^{d, k}(t)$.

Definition 6.3. In discrete riv-function $H^{d, k}=\left[h_{1}^{d, k}, h_{2}^{d, k}\right](\mathrm{d}$, k)-function $h_{1}^{d, k}(t) \quad\left(h_{2}^{d, k}(t)\right)$ we will be name the bottom (top) border of discrete riv-function $H^{d, k}(t)$.

Definition 6.4. For the set discrete riv-function $H^{d, k}=\left[h_{1}^{d, k}, h_{2}^{d, k}\right]$ set of all $(\mathrm{d}, \mathrm{k})$ - the functions $h^{d, k}(t)$, satisfying to a condition: for any $t$ where $1 \leq t \leq \mathrm{d}, \mathrm{d} \in \mathrm{N}^{+}$, $h_{1}^{d, k}(t) \leq h^{d, k}(t) \leq h_{2}^{d, k}(t)$, we will designate $\mathrm{W}\left(H^{d, k}(t)\right)$.

Definition 6.5. In discrete riv-function $H^{d, k}=\left[h_{1}^{d, k}, h_{2}^{d, k}\right]$ number of $(\mathrm{d}, \mathrm{k})$ - the functions $h^{d, k}(t)$, satisfying to a condition: for any $t$ where $1 \leq t \leq \mathrm{d}, \mathrm{d} \in \mathrm{N}^{+}, h_{1}^{d, k}(t) \leq h^{d, k}(t) \leq$ $h_{2}^{d, k}(t)$, we will designate $\Theta\left(H^{d, k}\right)$.

Definition 6.6. For (d, k) - function $h^{d, k}$ sequence < $p r_{2} h^{d, k}(1), \ldots, p r_{2} h^{d, k}(2), p r_{2} h^{d, k}(d)>$ of the second coordinates of pairs a kind $\left(\mathrm{t}, h^{d, k}(t)\right)$ where $1 \leq t \leq \mathrm{d}, \mathrm{d} \in \mathrm{N}^{+}$, we will designate $\xi\left(h^{d, k}\right)$.

(d, k) -function of kind $h^{d, k}$, because the range of definition is linearly ordered, is unequivocally defined by corresponding sequence of the second coordinates of pairs a kind (value of argument, value of function), i.e. sequence of a kind $\xi\left(h^{d, k}\right)=<p r_{2} h^{d, k}(1), \ldots, p r_{2} h^{d, k}(2), p r_{2} h^{d, k}(d)>$. Therefore the task of discrete riv-function $H^{d, k}=\left[h_{1}^{d, k}, h_{2}^{d, k}\right]$ is equivalent to definition of function of a kind $H^{d, k}=\left[\xi\left(h_{1}^{d, k}\right), \xi\left(h_{2}^{d, k}\right)\right]$. 
Definition 6.7. For the set discrete riv-function $H^{d, k}=\left[h_{1}^{d, k}, h_{2}^{d, k}\right]$ set of all sequences of a kind $\xi\left(h^{d, k}\right)=<$ $p r_{2} h^{d, k}(1), \ldots, p r_{2} h^{d, k}(2), p r_{2} h^{d, k}(d)>$, satisfying to a condition: for any $t$ where $1 \leq t \leq \mathrm{d}, \mathrm{d} \in \mathrm{N}^{+}, p r_{2} h_{1}^{d, k}(t) \leq p r_{2} h^{d, k}(t) \leq$ $p r_{2} h_{2}^{d, k}(t)$, we will designate $\Xi\left(H^{d, k}\right)$.

Definition 6.8. For the set discrete riv-function $H^{d, k}(t)=\left[p r_{2} h_{1}^{d, k}(t), p r_{2} h_{2}^{d, k}(t)\right]$ and chosen $t$ where $1 \leq t \leq \mathrm{d}$, $\mathrm{d} \in \mathrm{N}^{+}$, value of size $\left(p r_{2} h_{2}^{d, k}(t)-p r_{2} h_{1}^{d, k}(t)+1\right)$ we will designate $\mathrm{v}\left(H^{d, k}(t)\right)$.

For set discrete riv-function $H^{d, k}=\left[h_{1}^{d, k}, h_{2}^{d, k}\right]$ and discrete (d, k)-function $h^{d, k}$, for which is satisfied the condition: for any $t$, where $1 \leq t \leq \mathrm{d}, \mathrm{d} \in \mathrm{N}^{+}, h_{1}^{d, k}(t) \leq h^{d, k}(t) \leq h_{2}^{d, k}(t)$, size $\mathrm{v}\left(H^{d, k}(t)\right)$, where $1 \leq t \leq \mathrm{d}, \mathrm{d} \in \mathrm{N}^{+}$, defines number of various values, which the element with number $t$ (i.e. element $\left.p r_{2} h^{d, k}(t)\right)$ can accept in sequence $\xi\left(h^{d, k}\right)=<p r_{2} h^{d, k}(1) \ldots$, $p r_{2} h^{d, k}(2) p r_{2} h^{d, k}(d)>$, satisfying to a condition: for any $t$, where $1 \leq t \leq \mathrm{d}, \mathrm{d} \in \mathrm{N}^{+}, p r_{2} h_{1}^{d, k}(t) \leq p r_{2} h^{d, k}(t) \leq p r_{2} h_{2}^{d, k}(t)$.

Theorem 6.1. Cardinality $\Theta\left(H^{d, k}\right)$ of set $\mathrm{W}\left(H^{d, k}\right)$ of discrete $(\mathrm{d}, \mathrm{k})$-functions, defined by discrete riv-function $H^{d, k}=\left[h_{1}^{d, k}, h_{2}^{d, k}\right]$, is equal $\prod_{t=1}^{d}\left(h_{2}^{d, k}(t)-h_{1}^{d, k}(t)+1\right)$.

The proof.

Whereas the task of discrete riv-function $H^{d, k}=\left[h_{1}^{d, k}, h_{2}^{d, k}\right]$ is equivalent to definition of function of a kind $H^{d, k}=\left[\xi\left(h_{1}^{d, k}\right), \xi\left(h_{2}^{d, k}\right)\right]$, where $1 \leq t \leq \mathrm{d}, \mathrm{d} \in \mathrm{N}^{+}$, value $\Theta\left(H^{d, k}\right)$ for discrete riv-function $H^{d, k}=\left[h_{1}^{d, k}, h_{2}^{d, k}\right]$ can be defined on the basis of the analysis of number of sequences of a kind $\xi\left(h^{d, k}\right)=<p r_{2} h^{d, k}(1) \ldots, p r_{2} h^{d, k}(2) p r_{2} h^{d, k}(d)>$, satisfying to a condition: for any $t$, where $1 \leq t \leq \mathrm{d}, \mathrm{d} \in \mathrm{N}^{+}, p r_{2} h_{1}^{d, k}(t) \leq$ $p r_{2} h^{d, k}(t) \leq p r_{2} h_{2}^{d, k}(t)$.

For everyone $t$ where $1 \leq t \leq \mathrm{d}, \mathrm{d} \in \mathrm{N}^{+}$, element $p r_{2} h^{d, k}(t)$ of the sequence $\xi\left(h^{d, k}\right)$, satisfying to a condition $p r_{2} h_{1}^{d, k}(t) \leq$ $p r_{2} h^{d, k}(t) \leq p r_{2} h_{2}^{d, k}(t)$, can accept following values $p r_{2} h_{1}^{d, k}(t)$, $p r_{2} h_{1}^{d, k}(t)+1, p r_{2} h_{1}^{d, k}(t)+2, \ldots, p r_{2} h_{2}^{d, k}(t)$, i.e. $v\left(H^{d, k}(t)\right)=$ $\left(p r_{2} h_{2}^{d, k}(t)-p r_{2} h_{1}^{d, k}(t)+1\right)$ various values. The general number in pairs not equivalent sequences of a kind $\xi\left(h^{d, k}\right)=<$ $p r_{2} h^{d, k}(1), \ldots, p r_{2} h^{d, k}(2) p r_{2} h^{d, k}(d)>$ of len $\mathrm{d}$ is defined as product on all $t$, where $1 \leq t \leq \mathrm{d}, \mathrm{d} \in \mathrm{N}^{+}$, of numbers $v\left(H^{d, k}(t)\right)$. It means, that number of various sequences of a kind $\xi\left(h^{d, k}\right)=<p r_{2} h^{d, k}(1), \ldots, p r_{2} h^{d, k}(2), p r_{2} h^{d, k}(d)>$, satisfying to a condition: for any $t$ where $1 \leq t \leq \mathrm{d}, \mathrm{d} \in \mathrm{N}^{+}$, $p r_{2} h_{1}^{d, k}(t) \leq p r_{2} h^{d, k}(t) \leq p r_{2} h_{2}^{d, k}(t)$, is defined by expression $\prod_{t=1}^{d} \mathrm{v}\left(H^{d, k}(t)\right)=\prod_{t=1}^{d}\left(p r_{2} h_{2}^{d, k}(t)-p r_{2} h_{1}^{d, k}(t)+1\right)$. Hence, value $\Theta\left(H^{d, k}\right)$ for the set discrete riv-function $H^{d, k}=\left[h_{1}^{d, k}, h_{2}^{d, k}\right]$ is defined by expression of kind $\Theta\left(H^{d, k}(t)\right)=\prod_{t=1}^{d}\left(h_{2}^{d, k}(t)-h_{1}^{d, k}(t)+1\right) . \square$

For example, capacity $\Theta\left(H^{d, k}\right)$ of set $\mathrm{W}\left(H^{d, k}\right)$ of the discrete $(\mathrm{d}, \mathrm{k})$-functions, defined by discrete riv-function $H^{d, k}=\left[h_{1}^{d, k}, h_{2}^{d, k}\right]$, where $\mathrm{d}=40, \mathrm{k}=9, h_{1}^{d, k}(t)$ - characteristic function for prime numbers, and the top border $h_{2}^{d, k}(t)$ is set by the first 40 digits of number $\pi$, makes more than $10^{27}$.

\subsection{Estimation of Complexity of Laws and Processes of Functioning of Automatons with Use of Descrete Riv-Functions}

The finite The In clause are considered the initial finite determined automatons of type of Mile of kind $\mathrm{A}_{\mathrm{s}_{0}}=\left(S, X, Y, \delta, \lambda, s_{0}\right)$, where $S$ - set of states, $X$ - set of input signals, $Y$ - set of output signals, and $\delta: S \times X \rightarrow S$ - next-state function, $\lambda: S \times X \rightarrow Y-$ output function, and $s_{0} \in S$ - an initial state of the automaton. The automaton function on steps in abstract integer non-negative time $t \in N^{+}$, according to dynamics equations: $\mathrm{s}(\mathrm{t}+1)=\delta(\mathrm{s}(\mathrm{t}), \mathrm{x}(\mathrm{t})), \mathrm{y}(\mathrm{t})=\lambda(\mathrm{s}(\mathrm{t}), \mathrm{x}(\mathrm{t}))$.

In the given work is used the apparatus of geometrical images of laws of functioning of the automatons, for the first time offered by V. A. Tverdohlebov in 1995г. and later developed in work [4]. Transformation of phase pictures to geometrical images of laws of functioning of the automaton, offered and developed by V. A. Tverdohlebov, has allowed to represent phase pictures by uniform mathematical structures broken lines with numerical coordinates of points. To V. A. Tverdohlebov is shown, that sequence of elements from the finite set, combined with linear order on set of input words, defines laws of functioning of the discrete determined dynamic system (automaton).

V. A. Tverdohlebov is offered and developed a method of synthesis of laws of functioning of the automaton on the set sequence (see, for example, [4, 5]). Entered discrete rivfunction $H^{d, k}=\left[h_{1}^{d, k}, h_{2}^{d, k}\right]$ represents the set $(\mathrm{d}, \mathrm{k})$-functions, which specificity because the range of definition $(\mathrm{d}, \mathrm{k})$ function, is linearly ordered, presented-function by sequences of the second coordinates of pairs a kind (argument of function, value of function). In the given work such sequences are considered as sequence of the second coordinates of points of geometrical images of initial automatons. Thus, discrete riv-function $H^{d, k}=\left[h_{1}^{d, k}, h_{2}^{d, k}\right]$ at chosen set $\mathrm{X}=\left\{\mathrm{x}_{1}, \mathrm{x}_{2}, \ldots, \mathrm{x}_{\mathrm{k}}\right\}$ of input signals of the automaton and a linear order $\omega_{1}$ on set $\mathrm{X}^{*}$ of input sequences defines geometrical images of family $\alpha=\left\{A_{s_{0_{i}}}\right\}_{i \in I}$ of initial automatons. (Other interpretation of set $\Xi\left(H^{n, m}(t)\right)$ of sequences, defined by discrete riv-function $H^{d, k}=\left[h_{1}^{d, k}, h_{2}^{d, k}\right]$ is possible also. Discrete riv-function $H^{d, k}=\left[h_{1}^{d, k}, h_{2}^{d, k}\right]$ as a 
whole defines behaviour of one finite determined automaton of type of Mile $\mathrm{A}=(\mathrm{S}, \mathrm{X}, \mathrm{Y}, \delta, \lambda)$. For set discrete rivfunction $H^{d, k}=\left[h_{1}^{d, k}, h_{2}^{d, k}\right]$ each sequence $\xi\left(h^{d, k}\right) \in \Xi\left(H^{d, k}\right)$ defines behaviour of the initial automaton $\left(\mathrm{A}, \mathrm{s}_{0}\right)$, where $s_{0} \in S$. Accordingly capacity of set of state so defined automaton is $\left.\Theta\left(H^{d, k}(t)\right)=\prod_{t=1}^{d}\left(h_{2}^{d, k}(t) \quad h_{1}^{d, k}(t)+1\right).\right)$

Definition 6.9. Family $\alpha=\left\{A_{s_{0_{i}}}\right\}_{i \in I}$ of the finite determined automatons of type of Mile, where $A_{s_{0_{i}}}=\left(S_{i}, X, Y, \delta_{i}, \lambda_{i}\right)$, $s_{0_{i}} \in S_{i},|X|=m$ and $|Y|=l$, which geometrical images are set by sequences of set $\Xi\left(H^{d, k}\right)$, defined by discrete riv-function $H^{d, k}=\left[h_{1}^{d, k}, h_{2}^{d, k}\right]$, we will designate $\Psi\left(H^{d, k}, m, l\right)$.

The number of automatons in family $\Psi\left(H^{d, k}, m, l\right)$ is $\Theta\left(H^{d, k}(t)\right)=\prod_{t=1}^{d}\left(h_{2}^{d, k}(t) \quad h_{1}^{d, k}(t)+1\right)$.

The detailed description of a method of synthesis of the automaton on sequence, and also a method of check of equivalence of states of the automaton by it geometrical image contains in the monography [4]. We will note only the basic moments of a method of synthesis of the automaton on sequence. If as the task of laws of functioning of the automaton is considered the sequence $\xi\left(h^{d, k}\right)=<p r_{2} h^{d, k}(1)$, $p r_{2} h^{d, k}(2), \ldots, p r_{2} h^{d, k}(d)>$, then its interpretation joins splitting of sequence $\xi$ on pieces of length $\mathrm{m}$, where $\mathrm{m}=|\mathrm{X}|$. Each piece at such splitting defines functioning of the automaton for a concrete state of memory. If set of conditions of the automaton is $S=\left\{s_{p}\right\}_{p \in X^{*}}$, and next-state function $\delta$ define by rules $\mathrm{s}_{0}=\mathrm{s}_{\varepsilon}$ and $\delta\left(\mathrm{s}_{\mathrm{p}}, \mathrm{x}\right)=\mathrm{s}_{\mathrm{px}}$, then function $\delta$ appears standard for all automatons with set of input signals $X$. Specificity of automatons is shown, that on infinite set of states for each automaton classes of equivalent states are allocated. At synthesis of laws of functioning of the automaton is essential the way of regularization of next-state functions $\delta$ of the automaton. Various ways of regularization of next-state functions of the automaton are possible: cyclic regularization, regularization in an initial state, state can generate by random generator (from set of possible states), etc. In a case, when $\frac{d}{|X|} \neq\left[\frac{d}{|X|}\right]$, where $|\mathrm{X}|$ - number of input signals of the automaton, and $\mathrm{d}$ - the length of sequence $<$ $p r_{2} h^{d, k}(1), p r_{2} h^{d, k}(2), \ldots, p r_{2} h^{d, k}(d)>$, (on which laws of functioning of the automaton are constructed), regularization is required and for function of output $\lambda$. In the given part of work at research of properties of families of the automatons, set by discrete riv-functions, regularization of next-state functions is carried out by all specified ways, and value of capacity of the set of input signals and length of initial pieces of sequences are chosen in such manner, that $\frac{d}{|X|}=\left[\frac{d}{|X|}\right]$, therefore regularization of $\lambda$ is not required. Let's consider regularization of next-state function $\delta$ of the automaton of type of Mile $A=(S, X, Y, \delta, \lambda)$, constructed on sequence $\xi$ of length $\mathrm{d}$, provided that $\frac{d}{m}=\left[\frac{d}{m}\right]$, where $\mathrm{m}=|\mathrm{X}|$. Let $\tau=\left[\frac{\left(\left[\frac{d}{m}\right]-1\right)}{m}\right]$ and $\mathrm{b}=\left(\left[\frac{d}{m}\right]-1\right)-\tau \cdot \mathrm{m}$. When $\frac{d}{m}=\left[\frac{d}{m}\right]$ constructed on sequence $\xi$ of length d automaton A has $\frac{d}{m}$ states (i.e. $\mathrm{n}=|\mathrm{S}|=\frac{d}{m}$ ) and for states $\mathrm{s}_{\tau+1}, \mathrm{~S}_{\tau+2}, \ldots, \mathrm{s}_{\mathrm{n}}$ nextstate function $\delta$ of automaton $A$ is necessary for regularization. And in a case, when $\tau \neq \frac{\left(\frac{d}{m}-1\right)}{m}$ (i.e. $\mathrm{b}>0$ ), for next-state-function of automaton $\mathrm{A}$ for a state $\mathrm{s}_{\tau+1}$ are certain values $\delta\left(s_{\tau+1}, x_{v}\right)$, where $1 \leq v \leq b$, and values $\delta\left(s_{\tau+1}, x_{\mathrm{j}}\right)$, where $b+1 \leq j \leq m$, is required to regularized.

Definition 6.10. A type of regularization of next-state function $\delta$ of the finite determined automaton of type of Mile $A=(S, X, Y, \delta, \lambda)$, where $|\mathrm{X}|=m$, constructed on numerical sequence $\xi$ lengths $\mathrm{d}$, at which:

a. At $\mathrm{b}=0 \delta\left(s_{i}, x_{j}\right)=\mathrm{s}_{1}$, where $\tau+1 \leq i \leq \mathrm{n}, 1 \leq j \leq \mathrm{m}$;

b. At $\mathrm{b}>0 \delta\left(s_{i}, x_{j}\right)=\mathrm{s}_{1}$, where $\tau+2 \leq i \leq \mathrm{n}, 1 \leq j \leq \mathrm{m}$, and $\delta\left(s_{\tau+1}, x_{v}\right)=s_{1}$, where $1 \leq \mathrm{v} \leq b ;$

let's name regularization in an initial state (or regularization of type 1 ).

Definition 6.11. A type of regularization of next-state function $\delta$ of the finite determined automaton of type of Mile $A=(S, X, Y, \delta, \lambda)$, where $|\mathrm{X}|=m$, constructed on numerical sequence $\xi$ lengths $\mathrm{d}$, at which:

a. At $\mathrm{b}=0 \delta\left(s_{i}, x_{j}\right)=\mathrm{s}_{\sigma}$, where $\tau+1 \leq i \leq \mathrm{n}, 1 \leq j \leq \mathrm{m}$, $\sigma=(((i-1) \cdot m+j) \bmod \mu)+1, \mu=\left[\frac{d}{m}\right]$

b. At $\mathrm{b}>0 \delta\left(s_{i}, x_{j}\right)=\mathrm{s}_{\sigma}$, where $\tau+2 \leq i \leq \mathrm{n}, 1 \leq j \leq \mathrm{m}$, $\sigma=(((i-1) \cdot m+j) \bmod \mu)+1, \mu=\left[\frac{d}{m}\right]$, and $\delta\left(s_{\tau+1}, x_{v}\right)=s_{\sigma_{1}}$, where $1 \leq \mathrm{v} \leq b, \sigma_{1}=((\tau \cdot m+j) \bmod \mu)+1$ and $\mu=\left[\frac{d}{m}\right]$;

let's name cyclic regularization (or regularization of type 2).

In paper are considered the specific families of automatons $\Psi\left(H^{d, k}, m, l\right)$, defined by discrete riv-functions of kind $H^{d, k}=\left[h_{1}^{d, k}, h_{2}^{d, k}\right]$, where $h_{1}^{d, k}=C_{1}, h_{2}^{d, k}=C_{2}, \mathrm{C}_{1}, \mathrm{C}_{2} \in \mathrm{N}^{+}$, and $\mathrm{C}_{2}-\mathrm{C}_{1}+1=l, l \geq 2$ and $\mathrm{m} \in \mathrm{N}^{+}, \mathrm{m} \geq 2$.

Theorem 6.2. Let $H^{d, k}=\left[h_{1}^{d, k}, h_{2}^{d, k}\right]$ - the discrete rivfunction, where $h_{1}^{d, k}=C_{1}, h_{2}^{d, k}=C_{2}, \mathrm{C}_{1}, \mathrm{C}_{2} \in \mathrm{N}^{+}, \mathrm{C}_{2}-\mathrm{C}_{1}+1=l$, $l \geq 2$ and $\mathrm{m} \in \mathrm{N}^{+}, \mathrm{m} \geq 2$, and for family of automatons $\Psi\left(H^{d, k}, m, l\right)$ the condition $l^{\mathrm{m}} \geq\left[\frac{d}{m}\right]$ is satisfied, then at any way of regularization of next-state function $\delta$ of the automaton in family $\Psi\left(H^{d, k}, m, l\right)$ reduced automaton (automatons) with the maximum number of states has no more than $\left[\frac{d}{m}\right]$ states. The estimation is achievable. 
The proof.

Let's prove the theorem statement under the following plan. Each automaton from the family of automatons $\Psi\left(H^{d, k}, m, l\right)$, defined by discrete riv-function $H^{d, k}$, before minimisation has $\left[\frac{d}{m}\right]$ states (the method of construction of the automaton by numerical sequence see, for example, in work [4]). In discrete riv-function $H^{d, k}$ we will allocate so much initial parts, how many in pairs various broken lines of length $m$, take places in a rectangle $l \times m$, i.e. $l^{m}$ parts. We will assume, that the geometrical image of the automaton from family $\Psi\left(H^{d, k}, m, l\right)$ begins a part of the broken line, consisting from sequentially located (and in any order, but exact one time) in pairs various $l^{m}$ broken lines of length $m$. Then in the table of outputs of the automaton, constructed on such broken line, will contain $l^{m}$ various columns, i.e. automaton states with numbers from 1 to $l^{m}$ are 1recognizable states, so also simply recognizable states. In a case, when the condition $l^{\mathrm{m}} \geq\left[\frac{d}{m}\right]$ is satisfied, all $\left[\frac{d}{m}\right]$ are 1recognizable, so also are simply recognizable states. Therefore, at any way of regularization of next-state function $\delta$ of the automaton in family $\Psi\left(H^{d, k}, m, l\right)$ the reduced automaton (automatons) with the maximum number of conditions has no more $\left[\frac{d}{m}\right]$ conditions. The estimation is achievable. $\square$

Let's consider a specific way of regularization of next-state function $\delta$ of the automaton of type of Mile $A=(S, X, Y, \delta$, $\lambda)$, constructed on sequence $\xi$ lengths $\mathrm{d}$, provided that $\frac{d}{m}=$ $\left[\frac{d}{m}\right]$, where $\mathrm{m}=|\mathrm{X}|$ and $l=|\mathrm{Y}|$. Let $\tau=\left[\frac{\left(\left[\frac{d}{m}\right]-1\right)}{m}\right]$ and $\mathrm{b}=$ $\left(\left[\frac{d}{m}\right]-1\right)-\tau \cdot \mathrm{m}$. In a case, when $l^{m} \geq \tau$, next-state function $\delta$ of the automaton for states $s_{1}, s_{2}, \ldots, s_{\tau}$ is defined by a standard rule (set of states of the automaton is necessary set $S=\left\{s_{p}\right\}_{p \in X^{*}}$, and next-state function $\delta$ of the automaton is defined by rules $\mathrm{s}_{1}=\mathrm{s}_{\varepsilon}$ and $\delta\left(\mathrm{s}_{\mathrm{p}}, \mathrm{x}\right)=\mathrm{s}_{\mathrm{px}}$, and for states $\mathrm{s}_{\tau+1}, \mathrm{~s}_{\tau}$ $+2, \ldots, s_{n}$ next-state function $\delta$ of the automaton $A$ is required for regularization. And, when $\tau \neq \frac{\left(\frac{d}{m}-1\right)}{m}$ (i.e. $\mathrm{b}>0$ ), for next-state function $\delta$ of the automaton A for a state $\mathrm{s}_{\tau+1}$ are certain values $\delta\left(s_{\tau+1}, x_{v}\right)$, where $1 \leq \mathrm{v} \leq b$, and values $\delta\left(s_{\tau+1}, x_{\mathrm{j}}\right)$, where $b+1 \leq j \leq m$, is required to regularization.

In a case, when $l^{m}<\tau$, next-state function $\delta$ of the automaton for states with numbers $1,2, \ldots, l^{m}$ also is defined by a standard rule, and for states with numbers $l^{m}+1, l^{m}$ $+2, \ldots,\left[\frac{d}{m}\right]-1,\left[\frac{d}{m}\right]$ next-state function $\delta$ of the automaton $\mathrm{A}$ is required to regularization. Let $\eta=l^{m}$ and $\tau_{1}=\min (\eta, \tau)$. In definition 6.12 is offered the specific way of regularization to next-state function.

Definition 6. 12. A way of regularization to next-state function $\delta$ of the finite determined automaton of type of Mile $A=(S, X, Y, \delta, \lambda)$, where $|\mathrm{X}|=m,|\mathrm{Y}|=l$, constructed on numerical sequence $\xi$ lengths $d$, at which

$$
\begin{aligned}
& \delta\left(s_{\tau_{1}+1}, x_{1}\right)=\mathrm{s}_{1} \& \delta\left(s_{\tau_{1}+1}, x_{2}\right)=\mathrm{s}_{1} \& \ldots \& \delta\left(s_{\tau_{1}+1}, x_{m}\right)=\mathrm{s}_{1} \& \\
& \& \delta\left(s_{\tau_{1}+2}, x_{1}\right)=\mathrm{s}_{1} \& \delta\left(s_{\tau_{1}+2}, x_{2}\right)=\mathrm{s}_{1} \& \ldots \& \delta\left(s_{\tau_{1}+2}, x_{m}\right)=\mathrm{s}_{2} \& \\
& \ldots \ldots \ldots \ldots \ldots \ldots \ldots \ldots \ldots \ldots \ldots \ldots \ldots \ldots \ldots \ldots \ldots \ldots \ldots \ldots \ldots \ldots \ldots \ldots \ldots \ldots \ldots \\
& \& \delta\left(s_{\tau_{1}+\eta}, x_{1}\right)=\mathrm{s}_{1} \& \delta\left(s_{\tau_{1}+\eta}, x_{2}\right)=\mathrm{s}_{1} \& \ldots \& \delta\left(s_{\tau_{1}+\eta}, x_{m}\right)=\mathrm{s}_{\eta} \& \\
& \& \delta\left(s_{\tau_{1}+\eta+1}, x_{1}\right)=\mathrm{s}_{1} \& \delta\left(s_{\tau_{1}+\eta+1}, x_{2}\right)=\mathrm{s}_{1} \& \ldots \\
& \ldots \& \delta\left(s_{\tau_{1}+\eta+1}, x_{m-1}\right)=\mathrm{s}_{2} \& \delta\left(s_{\tau_{1}+\eta+1}, x_{m}\right)=\mathrm{s}_{1} \& \\
& \ldots \ldots \ldots \ldots \ldots \ldots \ldots \ldots \ldots \ldots \ldots \ldots \ldots \ldots \ldots \\
& \left.\& \delta\left(s_{n}, x_{1}\right)=\mathrm{s}_{\eta} \& \delta\left(s_{n}, x_{2}\right)=\mathrm{s}_{\eta} \& \ldots \& \delta s_{n}, x_{m-1}\right)=\mathrm{s}_{\eta} \& \delta\left(s_{n}, x_{m}\right)=\mathrm{s}_{\eta}
\end{aligned}
$$

let's name regularization of type 3 .

The theorem 6.3 is contained the condition (is resulted at use of a specific way of regularization (a way 3 ) of next-state function $\delta$ of the automaton), sufficient for achievement of the maximum estimation of number of states in the reduced automaton from family $\Psi\left(H^{d, k}, m, l\right)$, equal $\left[\frac{d}{m}\right]$ states.

Theorem 6. 3. Let $H^{d, k}=\left[h_{1}^{d, k}, h_{2}^{d, k}\right]$ - discrete riv-function where $h_{1}^{d, k}=C_{1}, h_{2}^{d, k}=C_{2}, \mathrm{C}_{1}, \mathrm{C}_{2} \in \mathrm{N}^{+}, \mathrm{C}_{2}-\mathrm{C}_{1}+1=l, l \geq 2$, and $\mathrm{m} \in \mathrm{N}^{+}, \mathrm{m} \geq 2$, and for the family of automatons $\Psi\left(H^{d, k}, m, l\right)$, defined by riv-function $H^{d, k}$, is satisfied condition $\left(l^{m}\right)^{m} \geq\left[\frac{d}{m}\right]-l^{\mathrm{m}}-1$, then at a way 3 of regularization to next-state function $\delta$ of the automaton in family $\Psi\left(H^{d, k}, m, l\right)$ the reduces automaton (automatons) with the maximum number of states has no more, than $\left[\frac{d}{m}\right]$ states. The estimation is achievable.

The proof.

Let's prove the theorem statement under the following plan. Each automaton from the family of automatons $\Psi\left(H^{d, k}, m, l\right)$, defined by discrete riv-function $H^{d, k}$, before minimisation has $\left[\frac{d}{m}\right]$ conditions (the method of construction of the automaton on numerical sequence see, for example, work [4]). In discrete riv-function $H^{d, k}$ we will allocate so much initial parts, how many in pairs various broken lines of length $m$, take places in a rectangle $l \times m$, i.e. $l^{m}$ parts. We will assume, that the geometrical image of the automaton from family $\Psi\left(H^{d, k}, m, l\right)$ begins a part of the broken line, consisting from sequentially located (and in any order, but exact one time) in pairs various $l^{m}$ broken lines of length $m$. Then in the table of outputs of the automaton, constructed on such broken line, will contain $l^{m}$ various columns, i.e. automaton states with numbers from 1 to $l^{m}$ are 1- recognizable states, so also simply recognizable states. For a designation of a subset of set of states of the automaton, consisting of states with numbers $l^{m}+1, l^{m}+2, \ldots,\left[\frac{d}{m}\right]-1$, $\left[\frac{d}{m}\right]$, we will use symbol $\mathrm{S}^{\prime}$. We will consider a case, when 
$l^{\mathrm{m}}<\left[\frac{d}{m}\right]$, i.e. $S^{\prime} \neq \varnothing$, (the case, when the condition $l^{\mathrm{m}} \geq\left[\frac{d}{m}\right]$ is satisfied is considered in a theorem 2). Each condition $s^{\prime} \in S^{\prime}$ will be necessarily is 1 -equivalent to any condition from set $\left(S \backslash S^{\prime}\right)$ (since in the table of outputs of the automaton for each column with number $l^{m}+1, l^{m}+2, \ldots,\left[\frac{d}{m}\right]-1,\left[\frac{d}{m}\right]$ there will be an identical column with number from 1 to $l^{m}$ ). We will show, that at use of a way of regularization to nextstate function $\delta$ of the automaton all states from set $\mathrm{S}^{\prime}$ will be 2- recognizable and, besides, any conditions $\mathrm{s}$ and $\mathrm{s}^{\prime}$ where $s \in S, s^{\prime} \in S^{\prime}$, which are 1-equivalent, are recognizable conditions. Because any two conditions with numbers from 1 to $l^{\mathrm{m}}$ are recognizable, at use of a way 3 of regularization to next-state function $\delta$ of the automaton for any states $\mathrm{s}^{\prime}$ and $\mathrm{s}^{\prime \prime}$ from set $\mathrm{S}^{\prime}$, provided that $\left(l^{m}\right)^{m} \geq\left[\frac{d}{m}\right]-l^{\mathrm{m}}-1$, exists input signal $\mathrm{x} \in \mathrm{X}$, such, that, if $\mathrm{s}^{\prime} \neq \mathrm{s}^{\prime \prime}$, then the following condition is satisfied: $\delta\left(s^{\prime}, x\right)=s_{i_{1}} \& \delta\left(s^{\prime \prime}, x\right)=s_{i_{2}} \quad$, where $s_{i_{1}}, s_{i_{2}} \in\left(S \backslash S^{\prime}\right)$ and $s_{i_{1}} \neq s_{i_{2}}$. It means, that any two states $\mathrm{s}^{\prime}$ and $\mathrm{s}^{\prime \prime}$ from set $\mathrm{S}^{\prime}$ are 2- recognizable, so also simply recognizable. Besides, at use of a way 3 of regularization to next-state function $\delta$ of the automaton, provided that $\left(l^{m}\right)^{m} \geq$ $\left[\frac{d}{m}\right]-l^{\mathrm{m}}-1$, for any states $\mathrm{s}$ and $\mathrm{s}^{\prime}$ where $s \in S, s^{\prime} \in S^{\prime}$, exists sequence $p \in X^{*}$ of length 2, such, that the condition is satisfied $\delta(s, p)=s_{j_{1}} \& \delta\left(s^{\prime}, p\right)=s_{j_{2}}$, where $s_{j_{1}}, s_{j_{2}} \in\left(S \backslash S^{\prime}\right)$ and $s_{j_{1}} \neq s_{j_{2}}$. Because states $s_{j_{1}}, s_{j_{2}} \in\left(S \backslash S^{\prime}\right)$ are 1- recognizable, any two states $\mathrm{s}$ and $\mathrm{s}{ }^{\prime}$ where $s \in S, s^{\prime} \in S^{\prime}$, are 3recognizable and so also simply recognizable states. As a result is had, that all $\left[\frac{d}{m}\right]$ states from set of states $\mathrm{S}$ are in pairs not equivalent, that proves the theorem statement. $\square$

\subsection{Example of Estimation of Complexity}

Example 1. Whereas information on the real law of functioning of concrete complex discrete dynamic system has huge dimension, and the law is known only partially and is required the decision of additional problems on regularization it to completely set law, we will spend an illustration of the offered method of an estimation of complexity on an example, in which the top and bottom borders of riv-function are presented by known mathematical sequences. On fig. 1 are shown the example of concrete rivfunction $H_{1}^{d, k}=\left[h_{1}^{d, k}, h_{2}^{d, k}\right]$, where $\mathrm{d}=40, \quad \mathrm{k}=9, \quad h_{1}^{d, k}(t) \quad$ characteristic function for Fibonacci numbers, and the top border $h_{2}^{d, k}(t)$ is defined by the first 40 digits of number $\pi$.

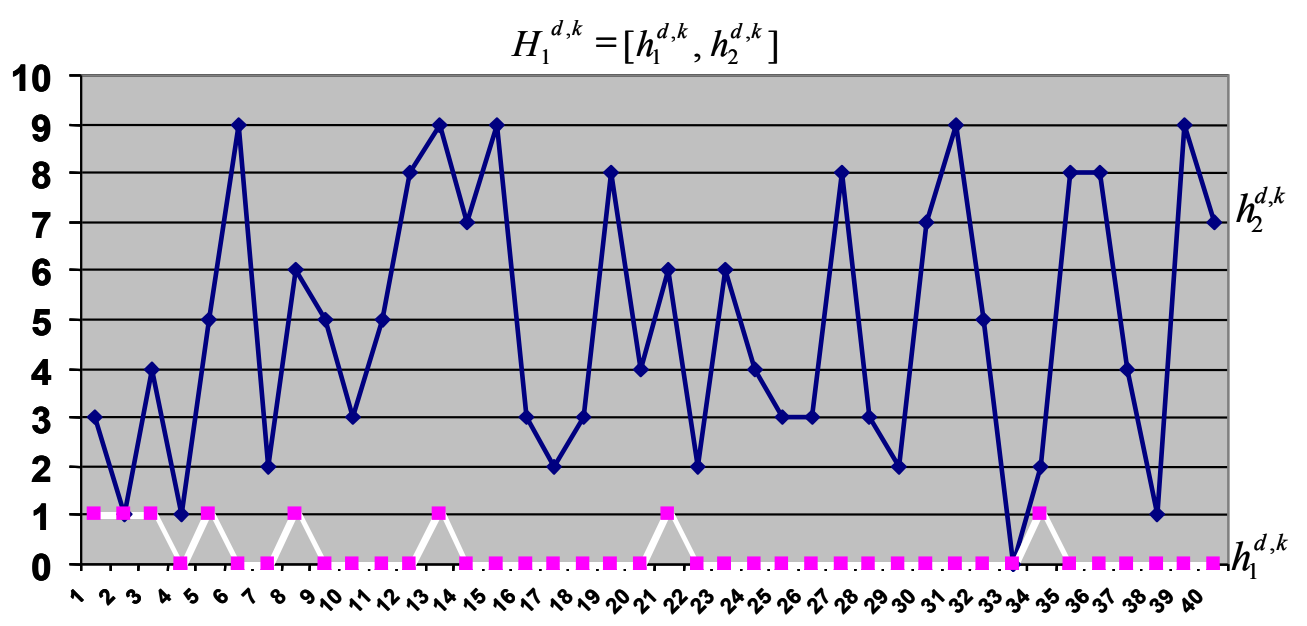

Figure 1. Image of discrete riv-function $H_{1}^{d, k}=\left[h_{1}^{d, k}, h_{2}^{d, k}\right]$.

On the basis of the spent analysis of riv-function, represented on fig. 1, are received concrete upper and lower estimates of number of states of automaton in the minimal form for all family of automatons (more than $10^{28}$ automatons), defined by this riv-function. In case, when geometrical image of automaton model of concrete discrete dynamic system is in the specified borders, then can specify precisely an interval of values for number of states in the minimal form of automaton model of system (accuracy to set of input signals of the automaton) without obviously constructing of the automaton and minimization. For this rivfunction and cardinality of set of input signals of the automaton equal, for example, 10, least number of states of the minimal form of the automaton equal two, and the greatest - 4 , i.e. at any of $10^{28}$ automatons (in the minimal form), defined by riv-function on fig. 1, cannot be less, than two states and cannot be more than 4 states.

Example 2. We will construct an estimation on the basis of the theorem 6. 3. Let $H^{d, k}=\left[h_{1}^{d, k}, h_{2}^{d, k}\right]$ - discrete riv-function, where $h_{1}^{d, k}=C_{1}, h_{2}^{d, k}=C_{2}, \mathrm{C}_{1}, \mathrm{C}_{2} \in \mathrm{N}^{+}, \mathrm{C}_{2}-\mathrm{C}_{1}+1=l$. We will consider a class of automaton, at which $|\mathrm{X}|=1000,|\mathrm{Y}|=320$, i.e. it is had $\mathrm{C}_{2}-\mathrm{C}_{1}+1=320$. We will admit, that length of an analyzed initial piece of geometrical images $\mathrm{d}=10^{10}$. Then the number of automaton in the family, defined by specified rivfunction, is equal $320^{10^{10}}$. On the basis of the theorem 3 we have, that the minimal automaton (automatons) with the greatest number of states has no more, than $10^{7}$ states. 


\section{Conclusion}

In given article on the basis of use of the apparatus of geometrical images of automatons are offered methods and the algorithms, developed for recognition of laws of functioning of discrete determined dynamic systems (automatons), set by the automaton mappings, placed on analytically set geometrical curves. Methods are proved by corresponding theorems. In paper are stated models and the methods developed for interpolation of partially set laws of functioning of automatons, set by the automata mappings placed on geometrical curves, using base points of the interpolation, selected on the basis of selection of autonomous subautomatons.

In paper is offered the estimation of complexity of laws of functioning of the discrete determined dynamic systems (automatons) on the basis of geometrical representation of laws and use of discrete riv-functions. Is carried out the analysis of more than 10 million the discrete riv-functions, formed by fundamental mathematical sequences of length to 80 signs, taken from bank [15]. Also are considered the rivfunctions, containing more of 20 billion of discrete graphs, on which laws of functioning of automatons are synthesised. Specificity of all considered riv-functions is defined. As complexity indicators are considered: $\mathrm{k}_{\min }$ - the minimum number of conditions at the reduced automaton in family $\Psi\left(H^{d, k}, m, l\right), \mathrm{k}_{\max }$ - the maximum number of conditions at the reduced automaton in family $\Psi\left(H^{d, k}, m, l\right)$. The number of states of modeling system is one of the fundamental characteristics, used at designing and system manufacturing. The offered method of an estimation of complexity of laws of functioning of the discrete determined automatons can be applied to get exact bottom and exact top estimations of number of states at the minimal automaton only on the basis of the analysis of a geometrical image of the automaton, without obvious construction of next-state and output functions of automaton and carrying out the subsequent minimization, which practical realization for automatons with large number of states even with use of modern computing systems is essentially complicated. The basic parameters, used in an offered method, are length of a considered initial piece of a geometrical image of the automaton - $d$, number of input signals of the automaton - $m$ and number of output signals of the automaton - 1 . In view of that the basic criteria for reception of estimations in an offered method are only ratios of sizes $\mathrm{d}, \mathrm{m}, \mathrm{l}$, and in a method recursive procedures of construction aren't used, the method can be used for the big finite sizes $d, m, 1$. Use of the apparatus of geometrical images of the automatons, offered and developed by V. A. Tverdokhlebov (see, for example, [4]), allows to consider geometrical curves and numerical sequences with automata interpretation, i.e. as ways of the task of laws of functioning of automatons. It allows to build automaton models of the discrete determined systems without restrictions on number of states. The offered method is based on use of geometrical representation of laws of functioning of automatons and allows to give concrete estimations on number of states for any, as is wished great, values of sizes $\mathrm{d}, \mathrm{m}, \mathrm{l}$, that can be used in practice at designing of systems for carrying out analysis on number of states of possible variants of realization of system for purpose of a choice of system with the least number of states.

The number of states of modeling system is one of the fundamental characteristics, used at designing and system manufacturing. The offered method of an estimation of complexity of laws of functioning of the discrete determined automatons can be applied to get exact bottom and exact top estimations of number of states at the minimal automaton only on the basis of the analysis of a geometrical image of the automaton, without obvious construction of next-state and output functions of automaton and carrying out the subsequent minimization, which practical realization for automatons with large number of states even with use of modern computing systems is essentially complicated. The basic parameters, used in an offered method, are length of a considered initial piece of a geometrical image of the automaton - $d$, number of input signals of the automaton - $m$ and number of output signals of the automaton - 1 . In view of that the basic criteria for reception of estimations in an offered method are only ratios of sizes $\mathrm{d}, \mathrm{m}, \mathrm{l}$, and in a method recursive procedures of construction aren't used, the method can be used for the big finite sizes $d, m, 1$. Use of the apparatus of geometrical images of the automatons, offered and developed by V. A. Tverdokhlebov (see, for example, [4]), allows to consider geometrical curves and numerical sequences with automata interpretation, i.e. as ways of the task of laws of functioning of automatons. It allows to build automaton models of the discrete determined systems without restrictions on number of states. The offered method is based on use of geometrical representation of laws of functioning of automatons and allows to give concrete estimations on number of states for any, as is wished great, values of sizes $\mathrm{d}, \mathrm{m}, 1$, that can be used in practice at designing of systems for carrying out analysis on number of states of possible variants of realization of system for purpose of a choice of system with the least number of states.

\section{References}

[1] E. Moore, "Gedanken-experiments on sequential machines", collection: Automata Studies, ed. C. E. Shannon and J. McCarthy [Russian translation], IL. 1956.

[2] A. Gill, "Introduction to the Theory of Finite-state Machines". McGraw-Hill, 1962.

[3] T. N. Hibbard, "Exact upper bounds for lengths of minimal experiments to determine the final state, for two classes of sequential machines", in: Cybernetics Collection, No. 2, In. Lit., Moscow, 1966.

[4] V. A. Tverdokhlebov, "The geometrical images of laws of functioning of finite state machines". Science book, Saratov, 2008.

[5] V. A. Tverdohlebov, A. S. Epifanov, "Representation of automatons by geometrical structures", Publishing Center Science, Saratov, 2013. 
[6] M. Sipser, "The history and status of the P versus NP question". Proceeding of 24th annual symposium on the theory of computing, 1994.

[7] R. Hofman, "Complexity considerations", cSAT lower bound. IMECS2007, Conference proceedings published by IAENG, 2007.

[8] M. Li, P. Vitanyi, "Introduction to Kolmogorov complexity and its applications". Springer-verilag, 1993.

[9] A. Borodin, I. Munro, "The computational complexity of algebraic and numeric problems". American Elsevier, New York, 1975

[10] H. Khalil, D. Ulery, "A review of current studies on complexity of algorithms for partial differential equations". ACM '76 proceedings of the 1976 annual conference, 1976.
[11] L. Fortnow, S. Homer, "A short history of computational complexity". Bulletin of the EATCS 80, 2003.

[12] Y. Kartynnik, A. Ryzhikov, "On minimum maximal distance-k matchings". Electronic notes in discrete mathematics, 2016, V. 56, PP. 71-76.

[13] V. Lozin, C. Purcell, R. Mosca, "Independent domination in finitely defined classes of graphs: polynomial algorithms", Discrete applied mathematics, 2015, V. 182, PP. 2-14.

[14] F. Foucaud, "Decision and approximation complexity for identifying codes and locating-dominating sets in restricted graph classes", Journal of discrete algorithms. 2015, V. 31, PP. 48-68.

[15] http://www.oeis.org. 